\title{
ON THE EXTENSION OF A THEOREM OF POINCARÉ FOR
}

\section{DIFFERENCE-EQUATIONS*}

BY

\author{
EDWARD B. VAN VLECK
}

One of the most useful theorems relating to difference equations is the wellknown theorem of PoIncaré $\dagger$ for the equation

$$
u(n+r)+P_{1}(n) u(n+r-1)+\cdots+P_{r}(n) u(n)=0,
$$

in which the coefficients $P_{i}(n)$ possess limits $A_{i}$ for $n=+\infty$. PoIncare showed, namely, that if the roots of the equation

$$
y^{r}+A_{1} y^{r-1}+\cdots+A_{r}=0
$$

are no two of equal modulus, the ratio $u(n) / u(n-1)$ has a limit for $n=+\infty$, and this limit is a root of (2), in general the root of greatest modulus. $\ddagger$

* Presented to the Society at the Chicago meeting, April 29, 1911.

† A merican Journal of Mathematics, vol. 7 (1885), p. 213. For careful analysis and further development of the theorem, see Perron, Crelle's Journal, vol. 136 (1909), p. 17 and vol. 137, p. 6.

$\ddagger$ In 1904, in an article on continued fractions in these Transactions, p. 253, I gave a proof of PoINCARE's theorem for $r=2$ which was suitable for application to the class of continued fractions there under consideration. This proof, and consequently its immediate applicability, was not fully understood by Pringsheim and called forth an interesting memoir from him (Sitzungsberichteder Bayerischen Akademie, math.-physikalischer Klasse, 1910, p. 3) devoted in large part to rederiving my results. Later after oral and written communication between us he has recognized the legitimacy of my proof (Ibid., 1911, p. 64). My argument was somewhat condensed, but not more so, it seemed to me, than is current in articles of an investigational character. A few additional lines, which it is worth while to add now, would have removed any difficulty.

When Pringsheim reaches the following theorem (p. 46), he inadvertently fails to attribute it to me, though the attribution is intended in the introduction:

If in the continued fraction

$$
A(z)=\frac{1}{1}+\frac{a_{2} z}{1}+\frac{a_{3} z}{1}+\cdots
$$

we have lim $a_{n}=k$ for $n=\infty$, the continued fraction will converge over the entire plane except (1) along the whole or a part of a rectilinear cut drawn from $z=-\frac{1}{1} k$ to $z=\infty$ with an argument equal to that of the vector from the origin to $z=-\frac{1}{4} k$, and except possibly (2) at certain isolated points $p_{1}, p_{2}, \cdots$. Within the plane so cut the continued fraction is holomorphic except at the points $p_{1}, p_{2}, p_{3}, \cdots$, which are poles. 
The equation (1) is obviously equivalent to the following linear system of first order:

$$
\begin{aligned}
& u_{1}(n+r)=-P_{1}(n) u_{1}(n+r-1)-P_{2}(n) u_{2}(n+r-1) \\
& u_{2}(n+r)=u_{1}(n+r-1), \\
& u_{3}(n+r)=u_{2}(n+r-1), \\
& \cdot \cdot \cdot \cdot \cdot \cdot P_{r}(n) u_{r}(n+r-1), \\
& u_{r}(n+r)=u_{r-1}(n+r-1) .
\end{aligned}
$$

In the following paper an analogue of PoINCARÉ's theorem is sought for the general linear system:

(A) $y_{i}(n)=a_{i 1}(n) y_{1}(n-1)+\cdots+a_{i r}(n) y_{r}(n-1) \quad(i=1,2, \cdots, r)$, in which the coefficients $a_{i j}(n)$ have limiting values $A_{i j}$ for $n=+\infty$. This extension, which is by no means superficially evident, is contained in Theorems 1 and 2 of $\S 2$.

When the coefficients of $(A)$ are constant, it gives for $n=1,2, \cdots$ the indefinite iteration of a collineation. This has led me to the following paper on the classification of collineations.

\section{\$1. Preliminary Considerations.}

Consider first the linear substitution $(A)$ for a fixed value of $n$ and seek the linear combinations of $y_{1}(n-1), \cdots, y_{r}(n-1)$ which in consequence of the substitution are altered only by a multiplicative constant; i. e.,

$$
\sum_{i=1}^{r} c_{i} y_{i}(n)=\rho \sum_{i=1}^{r} c_{i} y_{i}(n-1),
$$

Inserting here the values of the $y_{i}(n)$ given in $(A)$, we obtain an identity which gives the following system of equations for the determination of the ratios of the $c_{i}$;

$$
\begin{gathered}
{\left[a_{11}(n)-\rho\right] c_{1}+a_{21}(n) c_{2}+\cdots+a_{r 1}(n) c_{r}=0,} \\
a_{12}(n) c_{1}+\left[a_{22}(n)-\rho\right] c_{2}+\cdots+a_{r 2}(n) c_{r}=0, \\
\left.\cdot \cdot \cdot \cdot \cdot \cdot \cdot \cdot \cdot \cdot \cdot \cdot \cdot \cdot \cdot \cdot \cdot \cdot \cdot \cdot \cdot \cdot c_{r r}(n)-\rho\right] c_{r}=0 .
\end{gathered}
$$

The elimination of the $c_{i}$ gives for $\rho$ the familiar characteristic equation,

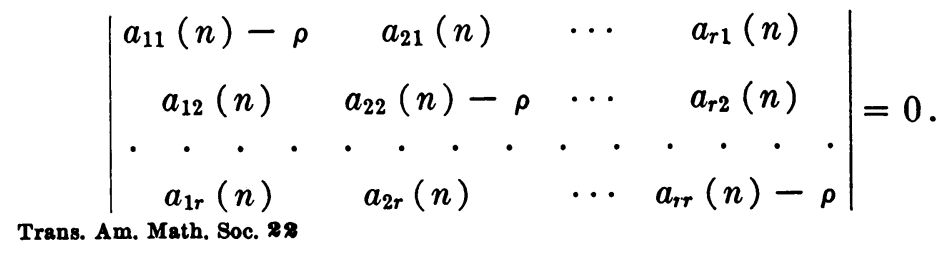


When $n$ increases indefinitely, every $a_{i j}(n)$ has by hypothesis a limit $A_{i j}$. The last equation has therefore the limiting form,

$$
\left|\begin{array}{ccccc}
A_{11}-\rho & A_{21} & \cdots & A_{r 1} \\
A_{12} & A_{22}-\rho & \cdots & A_{r 2} \\
\cdot & \cdot & \cdot & \cdot & \cdot \\
A_{1 r} & & A_{2 r} & \cdots & A_{r r}-\rho
\end{array}\right|=0 .
$$

Suppose now that no two roots $R_{i}$ of (7) are equal, and let them be arranged in the order of decreasing modulus,

$$
\left|R_{1}\right| \geqq\left|R_{2}\right| \geqq \cdots \geqq\left|R_{r}\right| \text {. }
$$

Then by taking $n$ sufficiently large the $r$ roots of $(6), \rho_{i}(n)(i=1, \cdots, r)$, may be distinguished from one another by virtue of their proximity to the roots $R_{i}$ of (7). Thus for a sufficiently large value of $N$ we will have

$$
\left|R_{i}-\rho_{i}(n)\right|<\frac{1}{2}\left|R_{i}-R_{j}\right| \quad(n>N ; j+i) .
$$

To each $\rho_{i}(n)$ there corresponds a set of equations (5), which we will now provide with appropriate subscripts and write:

$$
\left[a_{11}(n)-\rho_{i}(n)\right] c_{1 i}(n)+a_{21}(n) c_{2 i}(n)+\cdots+a_{r 1}(n) c_{r i}(n)=0,
$$

$$
a_{1 r}(n) c_{1 i}(n)+a_{2 r}(n) c_{2 i}(n)+\cdots+\left[a_{r r}(n)-\rho_{i}(n)\right] c_{r i}(n)=0 .
$$

From the theory of linear substitutions it is known that $r-1$ of these equations are independent when the roots of (6) are distinct.* These equations therefore completely determine the ratios of the $c_{j i}(n)(j=1, \cdots, r)$. For a like reason the limiting equations,

$$
\begin{gathered}
\left(A_{11}-R_{i}\right) C_{1 i}+A_{21} C_{2 i}+\cdots+A_{r i} C_{r i}=0, \\
\left.\cdot \cdot \cdot \cdot \cdot \cdot \cdot \cdot \cdot \cdot \cdot \cdot \cdot \cdot \cdot \cdot \cdot \cdot \cdot \cdot \cdot A_{r r}-R_{i}\right) C_{r i}=0,
\end{gathered}
$$

determine the ratios of the $C_{j i}$. Accordingly for each value of $i$ there is some one of the $C_{j i}$ which may be placed equal to 1 , and the ratios of the other $C_{j i}(j=1, \cdots, r)$ to this particular one will be finite. It follows then, since the coefficients of (10) are the limits of those in (9), that these ratios are the limits of the corresponding ratios of the $c_{j i}(n)$ for $n=\infty$. Hence if $N$ is chosen sufficiently large, the $c_{j i}(n)$ which correspond to the particular $C_{j i}$ taken equal to 1 , may also be set equal to 1 . We suppose hereafter that this has been done. By means of this convention the $c_{j i}(n)(j=1, \cdots, r)$ will be uniquely determined for $n \geqq N$, and accordingly we shall have $r$

* See, for example, pages 72-73 of HoRN's Gewöhnliche Differentialgleichungen beliebiger Ordnung. 
definite combinations,

$$
v_{i}(n)=\sum_{j=1}^{r} c_{j i}(n) y_{j}(n-1) \quad(i=1, \cdots, r),
$$

which possess the following property: When transformed by the linear substitution $(A)$, they undergo the multiplicative transformation

$$
\sum_{j=1}^{r} c_{j i}(n) y_{j}(n)=\rho_{i}(n) \sum_{j=1}^{r} c_{j i}(n) y_{j}(n-1) .
$$

For the limiting form of (11) we have

$$
V_{i}(n)=\sum_{j=1}^{r} C_{j i} Y_{j}(n-1) \quad(i=1, \cdots, r) .
$$

In the theory of linear substitutions* it is shown that the determinant $\left|c_{j i}(n)\right|$ can not vanish when the roots of $(6)$ are distinct. We may therefore solve (11) for the $y_{j}(n-1)$ in terms of the $v_{i}(n)$. Similarly the determinant $\left|C_{j i}\right| \neq 0$, and equations (12) may be solved for the $Y_{j}(n-1)$. The solutions will be denoted by

$$
\begin{aligned}
& y_{i}(n-1)=\sum_{j=1}^{r} d_{j i}(n) v_{j}(n) \quad(i=1, \cdots, r), \\
& Y_{i}(n-1)=\sum_{j=1}^{r} D_{j i} V_{j}(n),
\end{aligned}
$$

in which $D_{j i}$ is obviously the limit of $d_{j i}(n)$ for $n=\infty$.

The ratios of the $D_{j i}$ can be most easily determined by considering the linear system of difference equations with constant coefficients

$$
Y_{i}(n)=\sum_{j=1}^{r} A_{i j} Y_{j}(n-1) \quad(i=1, \cdots, r),
$$

to which the limiting relations (12) and (14) may be attached. For this system we have

$$
V_{i}(n+1)=R_{i} V_{i}(n) \text {. }
$$

Hence

$$
Y_{i}(n)=\sum_{j=1}^{r} D_{j i} V_{j}(n+1)=\sum_{j=1}^{r} D_{j i} R_{j} V_{j}(n) .
$$

If the expressions for $Y_{i}(n-1)$ and $Y_{i}(n)$ which are given in (14) and (16) are substituted in (15), and the coefficients of each $V_{j}(n)$ on the two sides of the equation are then equated, we obtain the following $r$ sets of relations

*Cf. HorN, loc. cit., p. 73. 
$(j=1, \cdots, r)$ :

$$
\begin{aligned}
& \left(A_{11}-R_{j}\right) D_{j 1}+A_{12} D_{j 2}+\cdots+A_{1 r} D_{j r}=0, \\
& A_{21} D_{j 1}+\left(A_{22}-R_{j}\right) D_{j 2}+\cdots+A_{2 r} D_{j r}=0 \text {, } \\
& A_{r 1} D_{j 1}+A_{r 2} D_{j 2}+\cdots+\left(A_{r r}-R_{j}\right) D_{j r}=0 .
\end{aligned}
$$

This shows that the $r$ coefficients $D_{j i}(i=1, \cdots, r)$ are the homogeneous coördinates of one of the invariant points of the linear substitution (15).

In considering the effect of the indefinite increase of $n$ upon any solution $y_{i}(n)(i=1, \cdots, r)$ of $(A)$, I propose to deal with them through the $v_{i}(n)$. For this purpose it will be necessary to find the relations subsisting between the $v_{i}(n+1)$ and $v_{i}(n)$. With the aid of $(A)$ we obtain

$$
\begin{array}{r}
v_{i}(n+1)=\sum_{j=1}^{r} c_{j i}(n+1) y_{j}(n)=\sum_{j=1}^{r} \sum_{k=1}^{r} c_{j i}(n+1) a_{j k}(n) y_{k}(n-1) \\
=\sum_{j=1}^{r} \sum_{k=1}^{r} c_{j i}(n) a_{j k}(n) y_{k}(n-1)+\sum_{j=1}^{r} \sum_{k=1}^{r}\left[c_{j i}(n+1)\right. \\
\left.-c_{j i}(n)\right] a_{j k}(n) y_{k}(n-1) .
\end{array}
$$

Now by virtue of (9) we have

$\sum_{k=1}^{r} \sum_{j=1}^{r} c_{j i}(n) a_{j k}(n) y_{k}(n-1)=\sum_{k=1}^{r} \rho_{i}(n) c_{k i}(n) y_{k}(n-1)=\rho_{i}(n) v_{i}(n)$.

As, furthermore, $\rho_{i}(n)$ has for $n=\infty$ the limit $R_{i}$ and $c_{j i}(n+1)-c_{j i}(n)$ has the limit 0 , equations (18) may be written,

$$
v_{i}(n+1)=R_{i} v_{i}(n)+\sum_{k=1}^{r} e_{k i}^{\prime} y_{k}(n-1),
$$

where $\lim e_{k i}^{\prime}(n)=0$ for $n=\infty$. Finally, if we substitute for the $y_{k}(n-1)$ their values in terms of the $v_{j}(n)$ as indicated in (13) and remember that the $d_{j i}$ have limits for $n=\infty$, we obtain, at least for $n>N$, the system of equations,

$$
v_{i}(n+1)=R_{i} v_{i}(n)+\sum_{j=1}^{r} e_{i j}(n) v_{j}(n) \quad(i=1, \cdots, r)
$$

where

$$
\lim _{n=\infty} e_{i j}(n)=0 \text {. }
$$

This system of equations forms the basis of the subsequent work. The values of the $y_{i}(n)$ and hence of the $v_{i}(n+1)$ depend upon the initial values given to the $y_{i}(0)$ and also upon the coefficients $a_{i j}(p),(p=1, \cdots, n)$. By exception the $y_{i}(n)$ and $v_{i}(n+1)$ may all vanish for some values of $n$, 
and consequently for all succeeding values. This can happen only if the determinant of the coefficients $a_{i j}(n)$ vanishes for at least one value of $n$. We shall assume in $\S 2$ that at least one of the $v_{i}(n)$ does not vanish for an infinity of values of $n$.

\section{§2. Extension of Poincarê's theorem.}

It will be supposed henceforth that

$$
\left|R_{1}\right|>R_{2}|>\cdots>| R_{r} \mid \text {. }
$$

When $n$ increases indefinitely, some one of the $r$ moduli $\left|v_{i}(n)\right|$ will be as large or larger than the remaining $r-1$ for an infinite number of values of $n$. I will show that this $\left|v_{i}(n)\right|$ will become and remain dominant over all the other $r-1$ moduli.

Suppose first that for an infinite set of values of $n$ we have

$$
\left|v_{1}(n)\right| \geqq\left|v_{i}(n)\right| \quad(i=2,3, \cdots, r),
$$

and denote this set by $\left\{n^{\prime}\right\}$. The division of the first of equations $(B)$ by $v_{1}(n)$ and of the succeeding equations by $v_{1}(n+1)$ gives

and

$$
\frac{v_{1}(n+1)}{v_{1}(n)}=R_{1}+e_{1}(n)
$$

$$
\frac{v_{i}(n+1)}{v_{1}(n+1)}=\frac{R_{i}}{R_{1}+e_{1}(n)} \cdot \frac{v_{i}(n)}{v_{1}(n)}+\frac{e_{i}(n)}{R_{1}+e_{1}(n)} \quad(i=2, \cdots, r),
$$

in which the $e(n)$ satisfy for $n=n^{\prime}$ the inequalities

$$
\left|e_{1}\left(n^{\prime}\right)\right| \leqq r \bar{e}\left(n^{\prime}\right), \quad\left|e_{i}\left(n^{\prime}\right)\right| \leqq r \bar{e}\left(n^{\prime}\right),
$$

where $\bar{e}\left(n^{\prime}\right)$ denotes the largest of the $\left|e_{i j}\left(n^{\prime}\right)\right|$ in $(B)$. Since $\lim \bar{e}\left(n^{\prime}\right)=0$ for $n^{\prime}=\infty$, it follows that for a sufficiently large value of $n^{\prime}$ we have

$$
\frac{\left|v_{i}\left(n^{\prime}+1\right)\right|}{\left|v_{1}\left(n^{\prime}+1\right)\right|} \leqq \frac{\left|R_{i}\right|}{\left|R_{1}\right|}+a,
$$

where $a$ is an arbitrarily prescribed positive quantity subject to the condition $\left|R_{i}\right| /\left|R_{1}\right|+a<1$. Consequently $n^{\prime}+1$ will be included with $n^{\prime}$ in the set $\left\{n^{\prime}\right\}$, provided only $n^{\prime}$ is taken sufficiently large. It follows that equations (20) to (23) will hold without exception from and after some sufficiently large value of $n$.

From (21) we now obtain

$$
\lim _{n=\infty} \frac{v_{1}(n+1)}{v_{1}(n)}=R_{1} .
$$


since $\lim \bar{e}(n)=0$. It may next be shown that

$$
\lim _{n=0} \frac{v_{i}(n)}{v_{1}(n)}=0 . \quad(i=2,3, \cdots, r) .
$$

For if this is not true, there will be at least some one value of $i$ and some fixed magnitude $M>0$ such that for an infinity of values of $n$ one has

$$
\frac{\left|v_{i}(n)\right|}{\left|v_{1}(n)\right|} \geqq M
$$

Call this set of values $\left\{n^{\prime \prime}\right\}$. Then since $\lim e_{1}(n)=\lim e_{i}(n)=0$, equation (22) for $n=n^{\prime \prime}$ may be written

$$
\frac{v_{i}\left(n^{\prime \prime}+1\right)}{v_{1}\left(n^{\prime \prime}+1\right)}=\left(\frac{R_{i}}{R_{1}}+e_{i}^{\prime}\left(n^{\prime \prime}\right)\right) \frac{v_{i}\left(n^{\prime \prime}\right)}{v_{1}\left(n^{\prime \prime}\right)},
$$

where $\lim e_{i}^{\prime}\left(n^{\prime \prime}\right)=0$ for $n^{\prime \prime}=\infty$. Since $\left|R_{i}\right|<\left|R_{1}\right|$, this shows that when $n^{\prime \prime}$ is sufficiently large, the ratio $\left|v_{i}\left(n^{\prime \prime}+j\right)\right| /\left|v_{1}\left(n^{\prime \prime}+j\right)\right|$ for $j=1,2, \cdots$ will decrease in approximately the ratio $\left|R_{i}\right|:\left|R_{1}\right|$ until a value of $j$ is reached for which the ratio falls below $M$. Thus if $\left|v_{i}(n)\right| /\left|v_{i}(n)\right|$ is equal to or greater than $M$ for an infinity of values of $n$, there must also be an infinity of such values for which it falls below $M$. But when it falls below $M$, equation (22) gives

$$
\frac{\left|v_{i}(n+1)\right|}{\left|v_{1}(n+1)\right|}<\frac{\left|R_{i}\right|}{\left|R_{1}+e_{1}(n)\right|} M+\frac{\left|e_{i}(n)\right|}{\left|R_{1}+e_{1}(n)\right|} .
$$

Since now $\lim e_{1}(n)=\lim e_{i}(n)=0$ for $n=\infty, n$ may be chosen so large in the last inequality that

$$
\frac{\left|v_{i}(n+1)\right|}{\left|v_{1}(n+1)\right|}<M
$$

Thus if $\left|v_{i}(n)\right| /\left|v_{1}(n)\right|<M$ for a sufficiently large value of $n$, it must remain so for all larger values of $n$. But this contradicts the hypothesis that . it exceeds $M$ for an infinity of values of $n$. Consequently (25) must hold, as was to be proved.

We shall next establish like results in case the $r-1$ inequalities

$$
\left|v_{2}(n)\right| \geqq\left|v_{i}(n)\right|
$$

hold simultaneously for an infinity of values of $n$. Denote this particular set of values by $\left\{n^{\prime}\right\}$. Then on dividing equations $(B)$ for $i=2$ by $v_{2}(n)$ and for $i \neq 2$ by $v_{2}(n+1)$ we obtain the following equations:

$$
\frac{v_{2}(n+1)}{v_{2}(n)}=R_{2}+e_{2}(n),
$$




$$
\begin{aligned}
& \frac{v_{1}(n+1)}{v_{2}(n+1)}=\frac{R_{1}}{R_{2}+e_{2}(n)} \cdot \frac{v_{1}(n)}{v_{2}(n)}+\frac{e_{1}(n)}{R_{2}+e_{2}(n)}, \\
& \frac{v_{i}(n+1)}{v_{2}(n+1)}=\frac{R_{i}}{R_{2}+e_{2}(n)} \cdot \frac{v_{i}(n)}{v_{2}(n)}+\frac{e_{i}(n)}{R_{2}+e_{2}(n)} \quad(i=3,4, \cdots, r),
\end{aligned}
$$

in which the $e(n)$ for $n=n^{\prime}$ satisfy the inequalities

$$
\left|e_{i}\left(n^{\prime}\right)\right| \leqq r \bar{e}\left(n^{\prime}\right)
$$$$
(i=1,2, \cdots, r) \text {, }
$$

where $\bar{e}\left(n^{\prime}\right)$ denotes the largest of the $\left|e_{i j}\left(n^{\prime}\right)\right|$ in $(B)$.

Equations (29) show that for a sufficiently large value of $n^{\prime}$ we have

$$
\left|v_{i}\left(n^{\prime}+1\right)\right|<\left|v_{2}\left(n^{\prime}+1\right)\right| \quad(i=3,4, \cdots, r)
$$

since

$$
\left|R_{i}\right|<\left|R_{2}\right|, \quad\left|v_{i}\left(n^{\prime}\right)\right| \leqq\left|v_{2}\left(n^{\prime}\right)\right|, \quad \lim _{n^{\prime}=\infty} \bar{e}\left(n^{\prime}\right)=0 .
$$

Furthermore, we must also have for a sufficiently large value of $n^{\prime}$

$$
\left|v_{1}\left(n^{\prime}+1\right)\right|<\left|v_{2}\left(n^{\prime}+1\right)\right|,
$$

for otherwise the inequality

$$
\left|v_{1}\left(n^{\prime}+1\right)\right| \geqq\left|v_{i}\left(n^{\prime}+1\right)\right| \quad(i=1,3, \cdots, r)
$$

would hold for an infinity of values of $n^{\prime}$. But the latter is impossible, since it has been shown that $\left|v_{1}(n)\right|$ would then ultimately become and remain dominant over all the other $\left|v_{i}(n)\right|$, which contradicts the hypothesis that (26) is to hold for an infinity of values of $n$. Now (31) and (32) together show that $n^{\prime}+1$ will be included simultaneously with $n^{\prime}$ in the set $\left\{n^{\prime}\right\}$, provided only $n^{\prime}$ is sufficiently large. It follows that (26)-(30) will all hold without exception from and after some fixed but large enough value of $n$.

From (27) we see next that

$$
\lim _{n=\infty} \frac{v_{2}(n+1)}{v_{2}(n)}=R_{2} \text {. }
$$

From (29) it follows also that

$$
\lim _{n=\infty} \frac{v_{i}(n+1)}{v_{2}(n+1)}=0 \quad(i=3, \cdots, r),
$$

the reasoning by which this is established being exactly parallel to that by which (25) was established on our former hypothesis. Lastly, it remains to be proved that

$$
\lim _{n=\infty} \frac{v_{1}(n)}{v_{2}(n)}=0
$$

Suppose, if possible, this not to be true. Then there must be some magnitude 
$M>0$ which $\left|v_{1}(n)\right| /\left|v_{2}(n)\right|$ will exceed for an infinity of values of $n$. Denote this set of values by $\left\{n^{\prime \prime}\right\}$. For these values (28) may be written

$$
\frac{v_{1}\left(n^{\prime \prime}+1\right)}{v_{2}\left(n^{\prime \prime}+1\right)}=\left(\frac{R_{1}}{R_{2}}+e^{\prime}\left(n^{\prime \prime}\right)\right) \frac{v_{1}\left(n^{\prime \prime}\right)}{v_{2}\left(n^{\prime \prime}\right)},
$$

where $\lim e^{\prime}\left(n^{\prime \prime}\right)=0$ for $n^{\prime \prime}=\infty$. Since $\left|R_{1}\right|>\left|R_{2}\right|$, this shows that ultimately $n^{\prime \prime}+1$ will be included with $n^{\prime \prime}$ in the set $\left\{n^{\prime \prime}\right\}$. Then as $i$ increases through the natural number system, the ratio $\left|v_{1}\left(n^{\prime \prime}+i\right)\right| / \mid v_{2}\left(n^{\prime \prime}+i \mid\right)$ will increase successively by factors approximating closer and closer to $\left|R_{1}\right| /\left|R_{2}\right|$, and hence finally we will have $\left|v_{1}\left(n^{\prime \prime}+i\right)\right|>\left|v_{2}\left(n^{\prime \prime}+i\right)\right|$ from and after some fixed value of $i$. But this contradicts our hypothesis (26). Hence (35) must hold, as stated.

In similar manner we may treat successively for $j=3,4, \cdots, r$ the hypothesis that the $r-1$ inequalities

$$
\left|v_{j}(n)\right| \geqq\left|v_{i}(n)\right|
$$

hold for an infinity of values of $n$. On dividing $(B)$ by $v_{j}(n)$ and $v_{j}(n+1)$ we obtain three sets of inequalities similar to (27), (28), (29), which furnish like results. We reach thus the following conclusion:

THEOREM 1. If in the system of differential equations $(B)$ we have

and we suppose

$$
\lim _{n=\infty} e_{i j}(n)=0 \quad(i, j=1,2, \cdots, r)
$$

$$
\left|R_{1}\right|>\left|R_{2}\right|>\cdots>\left|R_{r}\right|,
$$

then as $n$ increases indefinitely, either by exception the $r$ quantities $v_{i}(n)$ will all vanish from and after some fixed value of $n$, or some one of them will ultimately dominate over the other $r-1$. If this be $v_{k}(n)$, then

and

$$
\lim _{n=\infty} \frac{v_{k+1}(n)}{v_{k}(n)}=R_{k}
$$

$$
\lim _{n=\infty} \frac{v_{i}(n)}{v_{k}(n)}=0
$$

We return now to our initial equations $(A)$. We have seen that in the relations

$$
y_{i}(n-1)=\sum_{j=1}^{r} d_{j i}(n) v_{j}(n) \quad(i=1, \cdots, r)
$$

the coefficients tend toward limiting values $D_{j i}$. The determinant $\left|D_{j i}\right|$ can not vanish since it is the reciprocal of $\left|C_{j i}\right| \neq 0$. Consequently for each value of $j$ not all of the $D_{j i}(i=1, \cdots, r)$ can vanish. Consider now 
the equation

$$
\frac{y_{i}(n)}{y_{i}(n-1)}=\frac{\sum_{j=1}^{r} d_{j i}(n+1) v_{j}(n+1)}{\sum_{j=1}^{r} d_{j i}(n) v_{j}(n)}
$$

If, as in Theorem $1, v_{k}(n)$ denotes that one of the $v_{i}(n)$ which ultimately becomes dominant, and if $D_{k l}$ denotes one of the $D_{k j}(j=1, \cdots, r)$ which does not vanish, it follows by application of the theorem to (36) that

$$
\lim _{n=\infty} \frac{y_{l}(n-1)}{y_{l}(n)}=\lim \frac{d_{k l}(n+1) v_{k}}{d_{k l}(n)} \frac{(n+1)}{v_{k}}=\lim \frac{v_{k}(n+1)}{v_{k}(n)}=R_{k} .
$$

Also we have

$$
\lim _{n=\infty} \frac{y_{i}(n)}{y_{l}(n)}=\lim \frac{d_{k i}(n+1) v_{k}(n+1)}{d_{k l}(n+1) v_{k}(n+1)}=\frac{D_{k i}}{D_{k l}} . \quad(i \neq l) .
$$

This shows that the point $y_{i}(n)(i=1, \cdots, r)$ for $n=\infty$ approaches as its limit one of the invariant points of (15). This result may be restated as follows:

Theorem 2. Let $(A)$ be a system of difference equations in which the coefficients $a_{i j}(n)$ have limiting values $A_{i j}$ for $n=\infty$. If the roots $R_{i}$ of the limiting characteristic equation ( 7 ) are no two of equal modulus, then unless by exception the $y_{i}(n)$ all vanish from and after some fixed value of $n$, there is at least one function $y_{l}(n)$ in the solution $y_{j}(n),(j=1, \cdots, r)$, for which we have

$$
\lim _{n=\infty} \frac{y_{l}(n)}{y_{l}(n-1)}=R_{k}
$$

where $R_{k}$ is one of the roots of (7).

If also $(A)$ is a sequence of non-singular collineations in $r$ homogeneous variables, which have for $n=\infty$ a limiting form, then any initial point $y_{1}(0)$, $\cdots y_{r}(0)$ by the successive application of the collineations will approach as a limit one of the invariant points of the limiting collineation (15).

The theorem of PoIncaré is an immediate consequence of the first part of this theorem, for by the very construction of (3) we have

$$
\lim _{n=\infty} \frac{u_{i}(n)}{u_{i}(n+1)}=\lim \frac{u_{i-1}(n)}{u_{i-1}(n-1)} \quad(i=2, \cdots, r) .
$$

To guard against misconception I will add in conclusion that not all of the $r$ ratios $y_{i}(n) / y_{i}(n-1)$ need have limits for $n=\infty$, and even if they have, not all of the limits need to be roots of (7). The truth of the latter 
statement will be obvious from a consideration of the special system,

$$
\begin{aligned}
& v_{1}(n)=R_{1} v_{1}(n-1), \\
& v_{2}(n)=e(n) v_{1}(n-1)+R_{2} v_{2}(n-1)
\end{aligned}
$$

in which $e(n)$ is an infinitesimal having the value

$$
\left(S_{1}-R_{2}\right) \frac{\bar{v}_{2}(n-1)}{v_{1}(n-1)} \quad\left(0<R_{2}<S_{1}<R_{1}\right)
$$

Here the limit of $v_{2}(n) / v_{2}(n-1)$ for the particular integral $v_{i}(n)=\bar{v}_{i}(n)$ is an arbitrarily chosen constant $S_{1}$.

UNIVERSITY of Wrsconsts. 